\title{
UNA RESPUESTA AL DESAFÍO DE CAMPBELL: \\ LÄ EVOLUCIÓN DE TÉCNICAS Y FENÓMENOS EN LAS TRADICIONES EXPERIMENTALES*
}

SERGIO F. MARTÍNEZ

INSTITUTO DE INVESTIGACIONES FILOSÓFICAS

UNIVERSIDAD NACIONAL AUTÓNOMA DE MÉxICO

\section{Introducción}

En un trabajo reciente (1990), Campbell trata de formular una respuesta a las acusaciones repetidas durante varias décadas en contra de proyectos de la naturalización de la epistemología y en particular del modelo evolucionista de la epistemología que él propone. Campbell acepta que las acusaciones tienen base, ya que los modelos evolucionistas de la historia de la ciencia son "epistemológicamente vacios": su descripción de la continuidad de las creencias, de la propagación diferencial de linajes, de la retención selectiva de variantes y de la especiación de comunidades de creyentes, son descripciones que pueden aplicarse tanto a la historia de las sectas religiosas como a la historia de la ciencia. Según Campbell, lo que hay que especificar, y en esto consiste su desafío, es el tipo de "conocimiento" que requiere explicarse por medio de los modelos evolucionistas del conocimiento. La respuesta de Campbell, a grandes rasgos, es la siguiente. El tipo de conocimiento que requiere explicación, el tipo de conocimiento característico de la ciencia está constituido en su totalidad por "inducciones incompletas".

Para Campbell estas inducciones incompletas designan los rasgos más característicos de las teorías científicas, su subjustificación y subdeterminación. Propóne a continuación que hablemos de "epistemologías seleccionistas" en lugar de."epistemologías evolucionistas", y que de acuerdo con lo sugerido por esta nueva terminología nos restrinjamos a tratar de explicar aquello que aparezca como "diseñado para adecuarse" (designed-to-fit). Ahora bien, para Campbell, los problemas de este tipo en la epistemolo-

* Esta investigación es parte de mi trabajo en los proyectos de investigación IN-600289, IN-600192 y IN-400794 de la DGAPA, UNAM. Le agradezco a Edna Suárez sus comentarios a una primera versión de este trabajo y haberme proporcionado el ejemplo que utilizo en la sección 5 . 
gía tienen que ver con los problemas de la adecuación de las creencias al referente.

Campbell se considera dentro de la tradición epistemológica de Descartes y Kant, en tanto que se asevera este tipo de adecuación para algunas creencias y al mismo tiempo se acepta como problemática. Cualquier intento por fundamentar este tipo de tradición epistemológica corre el riesgo de caer en un círculo vicioso, o en una búsqueda del fundamento ad infinitum. La teoría de la selección, según Campbell, permite formular una respuesta que no es fundamentalista. Lo que Campbell nos ofrece como respuesta, sobre la base de la teoría de la selección y de su interpretación del problema central de la epistemología desde esta perspectiva, es un complejo mecanismo sociológico de persuasión interna en las comunidades científicas, de retención de las creencias y del cambio conceptual que hace posible que en algunas tradiciones científicas los referentes de las creencias hayan participado en la selección de nuevos consensos con respecto a las creencias.

En resumen, lo que se ofrece es una teoría causal de la referencia que no está basada en una concepción clásica de la causalidad (formulada en términos de transferencia de energía, contigüidad y prioridad temporal), sino en el tipo de causalidad "indirecta" modelada en la teoría de la selección orgánica (y en la generalización que Campbell propone). Esta teoría causal de la referencia es la base de lo que Campbell llama el dogma seleccionista: para que una creencia tenga referencia competente el referente debe de participar (por muy indirecta y parcial que sea) en la selección de la creencia.

Sin embargo, como veremos en la sección siguiente, si se toma en serio que la teoría de la evolución por selección natural es causal en un sentido que no es el sentido clásico, la identificación del referente de una creencia no puede hacerse independientemente del contexto impuesto por los diferentes escenarios adaptativos en los que se identifica al referente. Tomar en serio la idea de que la teoría de la evolución por selección natural es causal, en un sentido no clásico, tiene implicaciones que de hecho no permiten que una respuesta como la de Campbell sea viable. La respuesta de Campbell no es satisfactoria, pero su desafío sigue en pie.

Después de hacer ver las dificultades de una respuesta como la de Campbell en la sección 2, pasaré a examinar brevemente en la sección 3 el concepto de tradición científica y de tradición experimental en particular. En la sección 4 hago una breve presentación del concepto de regla heurística y su generalización a lo que llamo procedimiento heurístico. Presento las características principales distintintivas de los procedimientos heurísticos y el sentido en el que puede decirse qué conjuntos de procedimientos heurísticos evolucionan. Los procedimientos heurísticos se constituyen en lo que llamo estructuras heurísticas, las cuales son características de la producción 
de conocimiento en las tradiciones experimentales. Las técnicas experimentales pueden verse como reglas heurísticas en este sentido generalizado de regla heurística (i.e. como "procedimientos heurísticos"). En las secciones 5 y 6 estudio el concepto de variación de técnicas y muestro cómo se satisface el criterio de la agregatividad de las variaciones en el caso de las técnicas experimentales. La estructura heuristica de las técnicas experimentales con este mecanismo agregativo de variación constituye un modelo evolucionista del crecimiento del conocimiento en tradiciones experimentales. A este modelo lo llamo el modelo evolucionista de técnicas y fenómenos. La sección 7 explora algunas implicaciones de la discusión anterior para un proyecto de naturalización de la epistemología basado en el uso de modelos evólucionistas. Muestro en particular cómo el modelo de la evolución de técnicas y fenómenos puede responder a una serie de objeciones formuladas en contra de modelos evolucionistas del conocimiento, y del modelo de Campbell en particular. La sección 8 es un breve resumen y una conclusión.

\section{El concepto no clásico de causalidad en biología evolucionista}

El "realismo hipotético" de Campbell se sustenta en el supuesto de que existe una red causal humeana que nos debería permitir identificar un objeto independientemente de la caracterización que damos de él en los diferentes contextos en los que "aparece". En la medida en que los objetos o individuos que juegan un papel en nuestras explicaciones pueden describirse por medio de una teoría de acción local regida por leyes universales, podemos identificar sin problemas sucesos y objetos a través de los diferentes contextos. La identificación de los diferentes individuos a través de contextos ocurre en términos de su historia causal. Sin embargo, como veremos en esta sección, si abandonamos la idea de que el mundo es humeano, no hay razón para pensar que esta manera de identificar individuos se aplique en los contextos adaptativos, cuya construcción es un requisito de las explicaciones seleccionistas. En el sentido pertinente, la biología contemporánea parece obligarnos a abandonar ese supuesto y por lo tanto parece tornar sumamente problemática la estrategia de Campbell.

En biología, las partes $u$ objetos de un organismo se identifican por sus funciones con valor adaptativo. La descomposición de un organismo en unidades funcionales es a la vez una propuesta acerca de cuáles son las partes del organismo. La consideración de las unidades funcionales implica que tenemos un conocimiento acerca de los efectos que esas partes tienen en otras, y cómo esos efectos contribuyen a caracterizar el organismo como un todo. Sin embargo, no todas las funciones o efectos son relevantes. Un problema central de la biología evolucionista es la distinción entre diferentes. tipos de efecto y, en particular, entre los efectos que son meras consecuencias 
y los efectos que son (o han sido) realmente el resultado de funciones con valor adaptativo. Solamente estas últimas juegan un papel causal en las explicaciones evolucionistas.

Por ejemplo, la función (propia) de las orejas, es decir, la función que desempeña un papel causal en las explicaciones, no es su posible uso para colgarse aretes, sino el aumento de la capacidad auditiva en los seres humanos. La posibilidad de colgarse aretes es una mera consecuencia de una adaptación. La determinación de la función adaptativa (y, por tanto, del objeto o la parte relevante) solamente puede darse en el contexto de un escenario adaptativo. La delimitación de este contexto requiere a su vez de la reconstrucción de las fuerzas selectivas que actúan sobre una población de individuos en interacción con su medio. Estos individuos tienen una cierta estructura interna que les permite replicarse, y existen restricciones que regulan localmente las interacciones entre ellos, pero la selección no se produce en un lugar en particular, no es un suceso localizable como tiene que serlo todo suceso en el contexto de una causalidad humeana. La selección es un suceso que ocurre en las poblaciones y cuya explicación es probabilística.

Precisamente porque la selección no puede caracterizarse por lo que pasa en el nivel de interacciones mecánicas entre los individuos, y entre los individuos y su medio, la descripción de un proceso de selección requiere de la reconstrucción de las fuerzas de selección en el contexto de un problema adaptativo. Esta reconstrucción de cuáles son las fuerzas de selección sólo tiene sentido en el contexto de un problema adaptativo, y depende de lo que sabemos o suponemos acerca de la historia de la vida. Esto lo formularé diciendo que las explicaciones seleccionistas dependen del contexto. Una fuerza o presión de selección no existe de manera independiente del problema con respecto al cual se caracteriza. Con respecto al problema de explicar por qué tenemos un corazón, por ejemplo, se hace necesario caracterizar las presiones de selección que condujeron al desarrollo de un sistema circulatorio en algunos animales. Pero es sólo en el contexto de un problema de adaptación específico que podemos indentificar presiones de selección. El corazón es parte de un sistema circulatorio que tiene la función (en ciertos animales) de mantener una determinada temperatura constante y una distribución apropiada de oxígeno en los tejidos corporales, lo cual confiere a esos animales ventajas adaptativas. Esto lo podemos resumir diciendo que el contexto en el cual se selecciona algo, en tanto que cumple una función $F$, no es indiferente respecto a por lo menos algunas de las diferentes maneras de describir ese algo. No es posible dar una explicación satisfactoria de la función del corazón, desde el punto de vista de la teoría darwiniana de la evolución, sin tener en cuenta el hecho de que el corazón es un órgano de cierto tipo que tiene una historia evolutiva específica, la cual es parte constituyente de la explicación. 
Ahora bien, la propuesta de Campbell se basa en lo que él llama "el dogma seleccionista": para que una creencia tenga referencia competente, el referente debe de participar - por muy indirecta o parcialmente que sea- en la selección de la creencia. Esto requiere que el referente pueda identificarse por medio de algún proceso causal, a través de todos los escenarios adaptativos pertinentes independientemente de cómo ese proceso causal se reconstruya en situaciones específicas. Esto es algo que parece escapar a los recursos conceptuales de la teoría de la evolución. Por supuesto, en el caso de la física hay una intuición realista muy fuerte que sugiere que la identificación de una partícula a través de posibles interacciones no presente problema. La intuición, sin embargo, recurre al supuesto de que podemos utilizar algo como una red causal humeana constituida por leyes de aplicación universal, las cuales nos permiten identificar sin problema la partícula a través de todas las interacciones posibles.

De poder sustentar esta intuición sería posible argüir que qua objeto físico los objetos biológicos son identificables a través de los diferentes contextos. Pero ¿cómo sustentar esa intuición? Es posible sustentarla en la mecánica clásica, pero por qué vamos a sustentarla en esa teoría, que sabemos que es falsa. Y si pensamos en sustentarla en la mecánica cuántica la intuición no encuentra apoyo. Los problemas de no localización e individuación característicos de los objetos cuánticos ponen en duda cualquier intento por sustentar esa intuición en la mecánica cuántica.

La idea de que podemos decidir si el referente participa en la selección de la creencia (y de una inducción incompleta en particular) independientemente de los contextos adaptativos es un resabio de una concepción de la causalidad que no tiene cabida en la teoría de la evolución. El dogma seleccionista de Campbell no tiene más base que una metafísica apropiada a la física clásica, pero no a la biología evolucionista.

\section{El cambio científico como producto de la interacción de tradiciones}

Campbell supone que el tipo de conocimiento y el tipo de cambio que hay que explicar como distintivo de la ciencia es el conocimiento y el cambio de teorías. A partir del "dogma seleccionista", el problema de la adecuación de los referentes se plantea entonces como el problema central. La identificación implícita de Campbell de lo distintivo de la ciencia como sistema de creencias, con características del conocimiento teórico, sigue los lineamientos de la concepción dominante en filosofía de la ciencia. Según esta concepción, el conocimiento científico se articula en teorías. El objetivo de la ciencia se supone, es la construcción de teorías, mientras que el trabajo experimental, está desde una perspectiva epistemológica, subordinado a la 
inserción de sus resultados en esquemas teóricos. ${ }^{1}$ Una serie de estudios recientes han estado dirigidos a cambiar esta situación. Gallison (1987) y Pickering (1984) escribieron algunos de los primeros análisis importantes sobre la historia de las comunidades de físicos experimentales en el siglo XX. Hacking (1983) y Cartwright (1983) trajeron a colación importantes consecuencias filosóficas de este cambio de perspectiva. La sociología del conocimiento, y en particular trabajos como el libro de Shapin y Schaffer (1985) explotan otras vertientes de este cambio de perspectiva. En los últimos años una serie de libros y artículos ha sido dedicada al desarrollo de este nuevo nicho en la historiografía y la filosofía de la ciencia.

No voy a entrar aquí en los detalles de la distinción entre diferentes tipos de tradiciones científicas. Me ocuparé sólo de la distinción entre tradiciones experimentales y teóricas en la ciencia, y esto de una manera muy esquemática. Elaboraré la distinción sólo en la medida que es suficiente para hacer ver el sentido en el que las tradiciones experimentales, a diferencia de las teóricas, pueden modelarse como poblaciones de procedimientos heurísticos.

Para empezar, una tradición científica consiste en una comunidad de científicos histórica y sociológicamente identificable por el uso de patrones distintivos de razonamiento y de fines compartidos. Para modelar evolutivamente una tradición (en este caso experimental) se requiere introducir cierta terminología. Hull (1988) utiliza la distinción entre replicadores e interactores que nos será útil. Un replicador es una entidad que transmite su estructura casi intacta en replicaciones sucesivas, mientras que un interactor es una entidad que interacciona como un todo cohesionado con su medio ambiente, de tal modo que esta interacción causa que la replicación (i.e. el proceso de generación de réplicas) sea diferencial. Utilizando su terminología, en la mayoría de las ocasiones se puede ver a los científicos como los interactores de una comunidad científica. Los replicadores, en cambio, pueden ser de diferentes tipos, por ejemplo teorías (o ideas), técnicas experi-

1 La persistencia de esta filosofía de la ciencia orientada a la teoría puede apreciarse claramente incluso en los críticos más severos de la concepción filosófica tradicional de la ciencia. Feyerabend ha mostrado y recalcado en una serie de trabajos que no hay reglas formales metodológicas identificables a lo largo de la historia de la ciencia (incluso si nos restringimos a los mejores ejemplos de lo que es la ciencia). De aquí él concluye que no hay un método de la ciencia. Lo único que Feyerabend podría concluir es que no hay un método formal en la ciencia, que es una conclusión interesante, pero que está muy lejos de su pretendida conclusión. Otro ejemplo significativo es Laudan. Su libro Science and Values (1984) es un estudio sobre el papel que desempeñan los valores cognoscitivos en la conformación de la racionalidad científica. Laudan no sólo supone que estos valores cognoscitivos pueden estudiarse y evaluarse en términos del proceso de la aceptación de teorías, sino que presupone que no hay algún tipo de racionalidad no teórica (i.e. que no se exprese a través de las tradiciones teóricas) que juegue un papel importante en la conformación de nuestros valores cognoscitivos. 
mentales o narrativas. Sin embargo, me parece que no necesitamos limitarnos a la idea de que los interactores son sólo los científicos. Esta idea parece apropiada para el caso de las tradiciones teóricas, pero no necesariamente es el caso de las tradiciones experimentales. Más adelante voy a argüir que las técnicas experimentales, así como otros procedimientos heurísticos utilizados en el planteamiento y solución de problemas (por los científicos y aparatos) pueden concebirse también como los interactores principales, por lo menos en ciertos casos o desde ciertas perspectivas del cambio científico.

Que una tradición sea históricamente identificable quiere decir que la comunidad en cuestión se renueva gradualmente (por medio del proceso o procesos típicos de replicación), y que a través de ese proceso de replicación de los componentes o miembros de la comunidad hay valores y metas de investigación que se mantienen relativamente constantes, o cambian lentamente. En este sentido, una tradición es también un linaje (véase Hull 1988, cap. 11). Los diferentes tipos de tradiciones tienen replicadores que son predominantes o exclusivos de ese tipo de tradición, como los patrones de razonamiento o las estructuras de reglas heurísticas, por ejemplo. Por otra parte, hay replicadores que son compartidos por todas las tradiciones, tales como libros. Igualmente, los diferentes tipos de tradiciones tienen interactores que les son típicos, como cierto tipo de instrumentos o procedimientos heurísticos, entre otros.

Una tradición teórica puede concebirse como una comunidad de científicos y teorías sostenidas por esta comunidad que son identificables histórica y sociológicamente a partir del hecho de que los científicos de la comunidad en cuestión son los interactores principales y esas teorías, los replicadores principales. Las tradiciones teóricas se caracterizan por su fin primordial: la construcción de teorías con poder predictivo y/o explicativo, que estén basadas en unos cuantos principios básicos consistentes y que sean lo más general posible. ${ }^{2}$ Esta búsqueda de la generalidad y la consistencia expresada en teorías unificadoras encaja con la búsqueda de la incorporación de la mayor cantidad posible de razonamiento matemático y lógico. Por ello, es natural que estas teorías globales se asocien muchas veces con un sistema de ecuaciones, como las ecuaciones de Maxwell (asociadas con la teoría del electromagnetismo). Estas teorías globales pueden distinguirse de los modelos o

2 Esta caracterización de una tradición teórica permite explicar ciertos rasgos distintivos de esas tradiciones pero no se pretende que permitan explicar muchos aspectos importantes desde un punto de vista filosófico asociado con el cambio de teorías en la ciencia.

Los rasgos distintivos de las tradiciones teóricas, búsqueda de generalidades en las explicaciones, uso de razonamiento matemático, etc., pueden entenderse como factores que promueven el objetivo central de la tradición: la búsqueda de explicaciones unificadoras de fenómenos y teorías. En este modelo las variantes teóricas se seleccionan a partir de criterios guiados por ese objetivo unificador de las explicaciones. 
leyes fenoménicas ampliamente usados tanto en las ciencias naturales como en las sociales, como instrumentos de predicción y de explicación de fenómenos y procesos. Dentro de una tradición teórica se da por supuesto que las leyes y modelos fenoménicos pueden y deben derivarse siempre en el marco de las grandes teorías para su legitimación como conocimiento científico.

En cambio, una tradición experimental puede concebirse como constituida por interactores y replicadores de varias maneras. Por un lado, los experimentalistas son interactores y las técnicas experimentales los principales replicadores; por otro lado, las poblaciones de técnicas experimentales y de procedimientos heuristicos utilizados en la construcción y estabilización de un fenómeno son tanto interactores como replicadores principales. El hecho de que las técnicas o procedimientos heurísticos no produzcan a los científicos o las reglas heurísticas, en la manera en que los genes producen los organismos, no es una objeción para este tipo de modelo. ${ }^{3}$ En la explicación seleccionista del funcionamiento del sistema inmune se da una situación similar. Ciertos linajes de células en el sistema inmune son tanto interactores como replicadores principales.

La construcción y estabilización de fenómenos es la finalidad epistemológica central en las tradiciones experimentales. Este proceso da como resultado el atrincheramiento de los fenómenos y las técnicas. Para caracterizar la noción de atrincheramiento, el concepto básico en mi caracterización de las tradiciones experimentales es necesario introducir también el concepto de "linaje de fenómenos". Un linaje consiste en una serie de fenómenos que han evolucionado a partir de un mismo fenómeno. La noción de "linaje" hace énfasis en el hecho de que un fenómeno no existe aisladamente, sino que debemos verlo como parte de una red compleja de dependencias históricamente construidas. Cuando introduzca en la sección 6 los diagramas de dependencia de técnicas, se ampliará este punto.

Un fenómeno está atrincherado en la medida en que forma parte y contribuye a la creación y estabilización de otros fenómenos. Este tipo de atrincheramiento no es el único, pero sí el único del que hablaremos en este trabajo. En la sección 6 examinamos este concepto; en la próxima sección elaboraré el sentido en el que una tradición experimental se distingue por el uso de patrones de razonamiento. Estos patrones de razonamiento constituyen lo que llamaré "estructuras de procedimientos heurísticos". Principio introduciendo un contraste importante entre algoritmos y reglas heurísticas.

3 Los genes son los replicadores típicos. Alguien como Dawkins considera que los genes son los únicos replicadores principales en la evolución orgánica, Hull (1988, cap. 12), sin embargo, muestra que es posible pensar en células o incluso organismos como replicadores, y que no es claro en qué sentido sólo los genes deben de considerarse como los replicadores principales. Véase también sección 7. 


\section{Reglas heurísticas vs. métodos formales}

Un algoritmo es un sistema preciso de instrucciones que puede programarse en un computador para dar la solución a cualquier ejemplo (caso) de un problema. Por el contrario, una regla heurística es un sistema de instrucciones que genera una propuesta correcta en algunos casos y no en otros. Una regla heurística se refiere por lo general a un cierto método que se utiliza para dar solución a un problema de una manera que no garantiza su solución o la precisión de la solución. La corrección de un algoritmo es independiente de la situación en la que se aplica.

Hay problemas muy simples de lógica y matemática (como derivar las inferencias tautológicas, por ejemplo) de los que se conjetura con muy buenas razones que son intratables. Un problema es intratable si la solución del problema involucra algoritmos que requieren una cantidad físicamente no disponible de tiempo para su ejecución. Por supuesto, las reglas heurísticas pueden ayudar a resolver un problema que no podría resolverse en un tiempo físicamente disponible por medio de algoritmos formales. Sin embargo, en ese caso no podemos estar seguros de que obtendremos siempre la respuesta correcta. Típicamente, las reglas heurísticas sólo generan una respuesta confiable, para ciertos casos, de un problema y no para otros. El dominio de insumos (inputs) para los que la regla heurística es muy eficiente lo llamaremos el dominio de acción óptimo (una definición que dependerá de fijar convencionalmente algún criterio de eficiencia y/o de confiabilidad estadístico).

El trabajo de Kahnevan y Tverski (véase por ejemplo la colección de ensayos Kahneman et al., 1982) fue central en la formación, en la década de los cincuenta, de la disciplina que ahora se conoce como psicología cognitiva experimental. La psicología cognitiva trata precisamente de las limitaciones en las capacidades cognitivas y de cómo los procesos mentales y los juicios e inferencias moldeados por estas limitaciones constituyen procedimientos heurísticos. Un trabajo más reciente que trata de desarrollar un modelo del razonamiento en términos de procedimientos heurísticos es el de Holland $e t$ al., 1986. Wimsatt en varios trabajos ( 1980 y 1986 a en especial) ha caracterizado y ha hecho ver la importancia del uso de procedimientos heurísticos en cuestiones del cambio científico. Wimsatt ha puesto énfasis en el hecho de que las fallas y los errores que resultan del uso de reglas heurísticas no son azarosos, sino sistemáticos, y que el estudio de esas fallas y errores es parte muy importante de aprender a razonar por medios heurísticos. Estos estudios son parte de la base empírica sobre la que me apoyo para considerar que la noción de procedimiento heurístico que he esbozado arriba está relativamente bien entendida y que tiene las características que le atribuyo en este trabajo. 
Llamaré procedimiento heurístico a cualquier procedimiento no algorítmico que tiene las características de una regla heurística mencionadas anteriormente pero, que al contrario del concepto usual de regla, incluye además el tipo de interacciones con, o entre, sistemas materiales que generan las adaptaciones distintivas de los procesos biológicos, así como de los procesos técnicos. Una técnica experimental, por ejemplo, comúnmente involucra el conocimiento y la manipulación de ciertas propiedades de sistemas materiales que constituyen lo que llamaremos la base material de la técnica. Una serie de procedimientos que tienen que llevarse a cabo para la consecución de un fin está secuencialmente estructurada si para lograr ese fin es preferible que esa aplicación se haga en un cierto orden. Por lo menos a veces, para algunas subsecuencias, su orden es importante para la aplicación exitosa de la serie de procedimientos. Muchas veces las reglas, y más en general los procedimientos heurísticos, se aplican secuencialmente. Si queremos calcular aproximadamente el volumen de agua contenido en un estanque, podemos calcular a "ojo de buen cubero" el volumen. Si no confiamos en nuestro "ojo de buen cubero", podemos descomponer el problema en dos problemas que por lo general nos puede dar una mejor aproximación que nuestra primera impresión puramente intuitiva. Por medio de alguna regla heurística (o con la ayuda de una cinta de medir y conocimientos básicos de geometría) calculamos la superficie promedio del estanque, y por medio de otra regla heurística (que involucra el conocimiento que podamos tener del tipo de estanque en cuestión), calculamos la profundidad del estanque; para encontrar el volumen del estanque sólo tenemos que multiplicar la superficie estimada promedio por la profundidad estimada.

Sobre todo en problemas más complejos, en el tipo de problemas que se resuelven en la ciencia en particular, muchas veces la descomposición del problema en subproblemas es más compleja y requiere de procedimientos heurísticos para efectuar la descomposición en problemas (reglas de reducción) y reglas para resolver cada uno de los problemas parciales (reglas de operación).

Dos procedimientos tienen dominios de acción óptimos traslapados con respecto a un cierto (tipo de) problema o fin. Cuando ambos procedimientos son alternativas que pueden utilizarse de manera eficiente para la consecución de un fin. Por ejemplo, si dos técnicas de purificación pueden ser utilizadas de manera aparentemente equivalente en un experimento, decimos que las técnicas se traslapan o que tienen dominios de acción óptimo traslapados. Otro ejemplo: la superficie del estanque la podemos estimar por varios medios. Algunos de esos medios son procedimientos que pueden darnos una estimación más exacta pero que requieren más pasos y más trabajo. Otros procedimientos son más rápidos pero menos precisos. Dos 
procedimientos, $A$ y $B$, que con respecto al problema que se considere son aproximadamente equivalentes (en precisión y/o tiempo o pasos de ejecución o, en general, respecto a un parámetro relevante en un problema) diremos que se traslapan. Si el problema de calcular el volumen del estanque está en el dominio de $A$ y en el dominio de $B$, con una eficiencia semejante, ese problema está en el traslape de los dominios de $A$ y $B$.

Dos procedimientos tienen dominios de acción óptimos traslapados si para ciertos problemas, en cierto paso de la solución, cualquiera de los dos procedimientos puede ser utilizado sin alterar las estrategias posteriores de la solución.

Para desarmar un radio primero necesitamos de un cierto tipo de desarmador, pero a veces podemos elegir entre varios desarmadores de nuestra caja de herramientas. Posteriormente necesitaremos otro tipo de desarmadores y de herramientas más finas para desarmar el mecanismo del dial, o para cambiar un transistor, por ejemplo. Así, los diferentes tipos de desarmadores pueden tener dominios traslapados de aplicación y también tener aplicación secuencial en la solución de un cierto tipo de problema.

Llamaremos estructura heurística a una colección de procedimientos heurísticos organizados alrededor de la tarea de resolver cierto tipo de problemas o de construir o diseñar un cierto tipo de objeto. Algunos de estos procedimientos pueden aplicarse secuencialmente, o pueden tener dominios de acción óptimos traslapados.

Nótese que este tipo de relación (de traslape o de aplicación secuencial) depende del tipo de problema con respecto al cual pensemos el uso de la estructura heurística. Es más, creo que el problema de la caracterización de una estructura heurística es, en buena parte, el problema de caracterizar un tipo de problema.

Las herramientas que se encuentran en la caja de herramientas de un plomero tienen que ver con el tipo de problemas que espera encontrarse, y las. herramientas en la caja de un electricistartienen que ver con el tipo de problemas que éste espera encontrarse. Muchas herramientas en las dos cajas son las mismas o similares, pero hay otras que van a estar en la caja del plomero y no en la del electricista, y viceversa.

La diferencia de contenido entre la caja de herramientas del plomero y la del electricista nos ayuda también a ver un punto importante para la discusión. Sería muy difícil explicar el tipo de estructura heurística que ejemplifica la caja de un plomero a menos que tengamos idea del tipo de problema al que puede enfrentarse. Una estructura heurística debe verse como una estructura de interacción entre estructuras materiales (entre herramientas en la caja y sistemas de plomería, por ejemplo). En este sentido la caracterización de lo que llamo una estructura heurística está íntima- 
mente ligada a la caracterización de un tipo de problema como un tipo de interacción importante desde cierto punto de vista. En el caso de las tradiciones experimentales, el tipo de interacciones importantes desde el punto de vista de la ciencia son aquellas interacciones, y por lo tanto aquellas estructuras heurísticas, que contribuyen o juegan un papel en el desarrollo de técnicas y finalmente en la construcción y estabilización de fenómenos. En la siguiente sección muestro por medio de un ejemplo cómo las técnicas experimentales pueden verse como estructuras heurísticas.

\section{La técnica de secuenciación de aminoácidos como estructura heurística}

Cada tipo de proteína tiene una composición distintiva de aminoácidos que se ordenan en una secuencia determinada. Ya que una proteína tiene varios cientos de aminoácidos en su secuencia característica, el problema de determinar el orden y tipo de cada uno de los aminoácidos en esa secuencia es bastante complejo. Para resolver el problema se descompone en una serie de problemas más simples. En primer lugar, si la proteína está formada por más de una cadena polipeptídica, el primer paso consiste en separarlas (mediante hidrólisis), para posteriormente proceder a determinar por separado la secuencia de cada una. Los pasos centrales de la técnica más utilizada consisten en cortar muestras de cada una de esas cadenas utilizando diferentes enzimas. Cada tipo de enzima corta una cadena solamente en el punto en el que se encuentran ciertos aminoácidos (por ejemplo, la enzima tripsina solamente corta donde hay un aminoácido del tipo de la tirosina o la arginina). Estos fragmentos son separados y se determina su composición y su secuencia. El procedimiento se repite utilizando otras enzimas que cortan en puntos distintos de la cadena. El ciclo se repite con las cuatro o cinco enzimas que por lo regular se utilizan para ello. Una comparación de los diferentes fragmentos permite establecer el orden de la secuencia total.

Nótese que el orden de los procedimientos es importante para el éxito de la técnica. Si se empezaran los ciclos de corte con enzimas antes de separar las diferentes cadenas polipeptídicas de la proteína, no se podría determinar el orden de los fragmentos en cada una de las cadenas, pues los datos de composición y secuencia de aminoácidos corresponderían a una mezcla de fragmentos de las dos cadenas. En este caso las técnicas de corte dependen (en el contexto de la técnica de secuenciación) de las técnicas de la separación de cadenas. Más abajo elaboro una caracterización general de la idea de dependencia de técnicas.

Nótese también que hay un cierto orden que por lo regular debe seguirse en la aplicación de las enzimas para maximizar la eficiencia de la técnica. Deben aplicarse primero las enzimas cuya acción es más específica, es decir, las que actúan solamente donde se encuentra un tipo de aminoácido. De 
esa manera se sabrá con precisión qué tipo de aminoácidos se localizan en los extremos de cada fragmento. Esta información permitirá precisar los resultados obtenidos con las enzimas menos específicas.

El mismo tipo de estructuras heurísticas ejemplificado arriba con la técnica de la secuenciación de proteínas puede encontrarse en el diseño de la estructura del campo visual, en el "alambrado" del genoma y en teorías del funcionamiento del cerebro, así como en las reglas heurísticas que guían el comportamiento (Holland et al., 1986; Edelman, 1987).

Una vez que Campbell desarrolla su teoría de la evolución de capacidades cognitivas como evolución de una estructura heurística (en nuestra terminología), para responder a su desafío, tiene todavía que reformular su dogma de que la ciencia progresa a través de mejoras en la competencia de las creencias con sus referentes. Nuestra respuesta a su desafío, sin embargo, no tiene que ir más lejos que lo que permite la explicación de la generación apropiada de variantes y la selección de estructuras heurísticas (el tema de la próxima sección). Mi respuesta al desafío de Campbell no va a consistir en tratar de explicar el tipo de creencias científicas tal y como éstas son estructuradas en teorías globales, como hipótesis generales o, como dice Campbell, "inducciones incompletas" (que presuponen "el realismo hipotético" como punto de partida metafísico). Acepto la existencia de una brecha epistemológicamente significativa entre lo que explica un modelo seleccionista de las capacidades cognitivas asociadas con estructuras heurísticas, y el cambio de teorías. Una respuesta al desafío de Campbell no requiere que mostremos cómo puede cerrarse esta brecha, a menos que asumamos, como lo hace Campbell, que el tipo de conocimiento que requiere explicación en términos de un modelo evolucionista es el conocimiento sistematizado en teorías.

\section{La evolución de las técnicas experimentales}

Tal y como hemos caracterizado una estructura heurística, es claro que las técnicas experimentales que se emplean para la construcción y estabilización de fenómenos constituyen una estructura heurística. Lo distintivo de las técnicas experimentales, como procedimientos heurísticos, es que tienen siempre un referente material concreto, la base material de la técnica, que es parte del proceso causal que culmina en la construcción de fenómenos.

Las tradiciones experimentales tienen el objetivo de construir y estabilizar fenómenos. De este objetivo se sigue un criterio claro de selección de técnicas experimentales. Se seleccionan las técnicas que promueven la estabilización del tipo de fenómenos que se considera que promueven los fines del programa alrededor del cual se articula la tradición. En el contexto de las tradiciones experimentales es relativamente fácil caracterizar el concepto pertinente de adecuación. Exactamente en analogía con el caso biológico, 
una técnica contribuye a la sobrevivencia de su linaje en la medida en que es más exitosa para reproducirse $y$, posteriormente, atrincherarse, mediante su uso en la construcción y estabilización de nuevos fenómenos. La reproducción exitosa de técnicas (y por lo tanto de fenómenos concretos), por lo menos en parte, tiene lugar fuera de la tradición experimental, y depende de factores socioeconómicos como costo y mercado. Estos factores no juegan un papel, por lo menos directamente, en las primeras etapas de la construcción y la estabilización de un fenómeno (pero sin embargo inciden en la adecuación de la tradición).

Es necesario introducir ahora una distinción importante. Cuando hablamos de técnicas nos referimos ambiguamente por lo general a técnicas concretas (tokens) y a técnicas tipo. Un ejemplo de técnica concreta es la técnica utilizada por el laboratorio del doctor perengano para la secuenciación de proteínas. ${ }^{4}$ Más precisamente, la técnica utilizada por el doctor perengano para la secuenciación de proteínas en su laboratorio de la UNAM el día 27 de junio de 1994. Nótese que esta caracterización de la técnica concreta no deja de ser ambigua. Una manera de tratar de evitar esa ambigüedad en la distinción entre técnicas concretas y técnicas tipo es recurriendo a una idealización de las técnicas individuales que extiende el proceso sugerido antes. Una técnica individual se va a caracterizar por llevarse a cabo en cierto lugar, en cierto tiempo. Un tipo de técnicas podría entonces caracterizarse como una clase de técnicas individuales caracterizadas como fenómenos espaciotemporales. Este tipo de caracterización de la distinción, sin embargo, no es satisfactorio.

En primer lugar, la solución anterior al problema de la distinción entre técnicas concretas o individuales y técnicas tipo no es satisfactorio porque la idealización en la que se basa no encaja con el tipo de uso al que sirve la distinción. La distinción sirve para hacer generalizaciones. Decir que la técnica llevada a cabo hoy en el laboratorio del doctor perengano es de tal tipo es decir algo respecto al tipo de fenómenos, errores o niveles de confiabilidad asociados con ese tipo de técnica. Decir que tuvo lugar en tal y tal lugar y en tal tiempo no tiene nada que ver con la generalización y, por lo

4 Una distinción similar ocurre en biología evolutiva. No es problemática la individuación de los diferentes organismos vertebrados, pero una clasificación similar en el caso de muchos organismos no vertebrados es bastante problemática, y una decisión al respecto es una parte importante de cualquier teoría evolucionista de esos organismos. El problema es todavía más obvio y paralelo al problema que aquí nos interesa en el caso de la ecología evolucionista. Un problema central de la ecología evolucionista es explicar la relación que existe entre clasificaciones de interacciones (y su distribución geográfica) con el concepto y la dinámica de las especializaciones de las diferentes especies, y en general con el concepto de coevolución. (Véase, por ejemplo, Jhon Thompson, The Coevolutionary Process, Chicago, 1995). Este tema lo he desarrollado más a fondo en Martínez, 1995b. 
tanto, hace misteriosa la generalización. En segundo lugar, la identificación de una técnica con un evento espacio-temporal deja a un lado el hecho de que una técnica individual no es algo simple, sin partes o sin estructura interna. Cada técnica individual tiene una estructura interna que hace que el proceso causal que sucede en un determinado espacio-tiempo sea identificable como técnica. Esta estructura interna no es caracterizable en términos de las propiedades "internas" de las partes, en términos de las propiedades inherentes de los materiales que entran en juego en el proceso causal que constituye la técnica individual. Las partes de una técnica individual, su estructura como tal, sólo pueden caracterizarse funcionalmente en relación con el tipo de técnicas del que pensemos que la técnica individual es parte. Reconocer esta caracterización funcional de las partes es importante para entender la manera como una técnica concreta es el punto de partida de generalizaciones (véase Martínez, 1995b). Sin embargo, de ahora en adelante no voy a marcar cuándo es posible la distinción entre técnicas concretas y técnicas tipo. Recurriré a la distinción sólo cuando la referencia para el problema en cuestión pueda ser ambigua. En general, voy a hablar de técnicas tipo.

Los mecanismos de variación del modelo de evolución de técnicas experimentales que me interesa desarrollar provienen de los diferentes sentidos y grados de libertad en los que las variantes de una técnica concreta son agregativas. Cada uno de los diferentes sentidos en los que una técnica es agregativa con respecto a sus partes, en relación con la construcción de un fenómeno, es una fuente de variabilidad.

Decimos que una técnica $T$, cuya función $F$ es la contrucción y estabilización de un fenómeno dado, es agregativa respecto a cierta descomposición en partes (inducida por F) en la medida en que las partes (pasos o subtécnicas) pueden sustituirse por otras técnicas cuyo origen y composición material pueden ser muy diferentes, pero cuya función es equivalente a la que sustituye, en relación con la función $F$ de $T$. En realidad, esta noción de agregatividad se refiere a una familia de tipos de agregatividad. Podemos distinguir, por ejemplo, el caso en el que dos subtécnicas de la misma técnica (concreta) son intercambiables, del caso en el que la técnica que se sustituye proviene de otra técnica (tipo). Ésta y otras distinciones no son importantes para nuestro propósito y no van a ser estudiadas con detalle. Sin embargo, en la próxima sección examinaremos, sobre la base de algunos ejemplos, tres tipos o fuentes de variabilidad que provienen de la distinción entre tres tipos de agregatividad (con respecto a una función). ${ }^{5}$

5 Para una caracterización bastante detallada de diferentes tipos de agregatividad, véase Wimsàtt, $1986 \mathrm{~b}$. 
¿Cuál es la razón por la que solamente las "variantes agregativas" cuentan como fuentes de variabilidad de la técnica? La agregatividad de una variante técnica indica su independencia con respecto a la organización de sus partes, esto es, aquello que se presenta como una "parte independiente" o función que está sujeta a la acción de la selección. El teorema fundamental de la selección natural propuesto en 1930 por Fisher tiene, entre otras consecuencias, la de que debe haber variación en la adecuación (debida a una alteración en una función) para que pueda haber un cambio evolutivo debido a la selección.

Creo que no está de más recalcar que no pretendo que exista un nivel último de agregatividad. Los diferentes sentidos de agregatividad no se refieren a las diferentes maneras de descomponer un sistema en sus partes cada vez más pequeñas o básicas. Una técnica se descompone en subtécnicas de diferentes maneras; la descomposición es relativa a una función. Una técnica de detección de un cierto tipo de sustancia, por ejemplo, puede ser suficientemente precisa para ciertos fines, pero no para otros. $\mathrm{Si}$, por ejemplo, queremos adaptar la técnica para un fin en el cual la precisión de la técnica en cuestión no es suficiente, vamos a buscar la manera de encontrar una variante que aumente su precisión.

La variabilidad puede depender (y esta dependencia constituye una restricción) de las necesidades o presiones sociales que inciden en los procesos de construcción de fenómenos. Va a depender también de las características de los materiales utilizados, de sus propiedades y de la manera como esas propiedades son pertinentes en el proceso de construcción de fenómenos. Por ejemplo, si un cierto instrumento es de hierro, debe de buscarse un sustituto en los casos en que esa propiedad del instrumento lo haga desventajoso. El costo de los instrumentos no es por lo general un factor crucial en el proceso de la construcción y estabilización de un fenómeno, pero sí es muy importante cuando se trata de incorporar el fenómeno al proceso tecnológico-industrial de la producción de fenómenos estabilizados.

El análisis de Wimsatt en $1986 \mathrm{~b}$ sobre las propiedades de los sistemas y sus partes analiza la agregatividad como un tipo de relación entre las propiedades de los sistemas y sus partes. El análisis de la agregatividad de técnicas que introduzco aquí como una modificación del análisis de Wimsatt requiere que enfoquemos nuestra atención en las propiedades de sistemas materiales en la medida en que éstas inciden en las funciones que desempeñan esos sistemas en el proceso de estabilización de un fenómeno. ${ }^{6}$

Una técriica $T$ está constituida de subtécnicas o pasos $t_{i}$ que dependen entre ellas de manera compleja; esta dependencia es una expresión de

6 Otros aspectos de este modelo de la evolución de técnicas y fenómenos se desarrollan en Martínez, 1995a; Martínez 1995b; Martínez y Suárez, 1996. 
la subordinación de las funciones propias de las diferentes subtécnicaspasos $t_{i}$ al objetivo de $T$ (representado por la función $F$ ). ${ }^{7}$ La dependencia puede entenderse de muchas maneras. Para la discusión presente es suficiente que entendamos esa dependencia en términos de las condiciones suficientes para la aplicabilidad de una técnica. Así, que $t_{i}$ sea dependiente de $t_{j}$ quiere decir que la aplicación de $t_{i}$ requiere, en el contexto de una técnica $T$ diseñada para generar un cierto fenómeno, la aplicación previa de $t_{i}$. Esto es, $t_{j}$ se aplica en un dominio que resulta de la aplicación de $t_{i}$.

El tipo más sencillo de dependencia de técnicas es el de una cadena, esto es, de una serie de técnicas cuya aplicación requiere de la aplicación antecedente de otra regla. Este caso puede representarse en un diagrama como sigue:

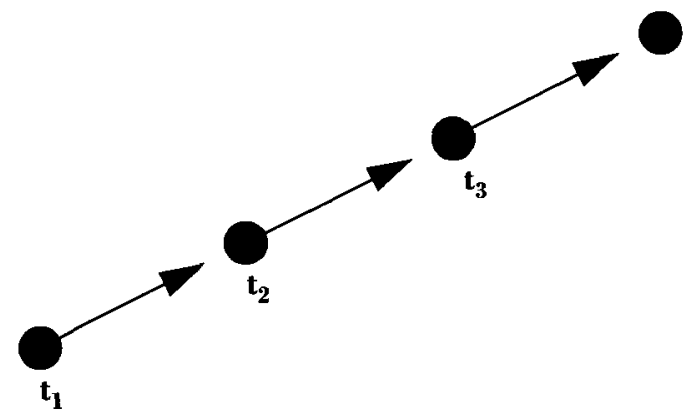

(Fig. 1) El diagrama describe una serie de pasos (técnicas) $t_{i}$ tal que una técnica a la derecha depende (para su aplicación en el contexto de alguna técnica $T$ del cual son subtécnicas) de todas las otras técnicas a la izquierda.

El digrama describe las dependencias (importantes en un problema específico) con respecto a un cierto fin (que siempre se entiende como la construcción y estabilización de algún fenómeno específico). ${ }^{8}$ Cada nodo

7 Una subtécnica $t_{i}$ es considerada un paso de $T$ en relación con una función $F$, pero con respecto a otra función $G$, puede ser parte de una técnica diferente, o puede descomponerse en diferentes subtécnicas TG. Usaré, en ocasiones, indistintamente las palabras "técnica" y "subtécnica", lo cual indica que el carácter de éstas se determina en un contexto funcional. Una técnica $T F$ es un conjunto de técnicas cuyo objetivo es la construcción de un fenómeno (concreto $F$ ). La función $F$ puede pensarse como el diseño del fenómeno, que a su vez puede identificarse con el fenómeno concepto asociado con la función $F$.

8 - Por supuesto, esta dependencia puede concretarse de diferentes maneras. Una técnica $A$ puede depender de otra técnica $B$ en el sentido đe que $B$ no puede llevarse a cabo si $A$ no está 
representa un paso de la técnica. Cada paso (subtécnica) es, por supuesto, una técnica por sí misma, esto es, una técnica que en un cierto momento podemos considerar como unitaria y simple. No cambia las cosas el hecho de que esa unidad sea relativa a la función o funciones que nos interesan en un determinado momento. Lo importante es que quede claro que, si bien con respecto a una cierta función $F$ (de una técnica $T$ de la que la cadena de técnicas anterior sería una subtécnica) las subtécnicas $t_{i}$ y $t_{j}$ (digamos) son intercambiables, es posible que no lo sean con respecto a otra función. Por ejemplo, para un cierto fin la pérdida de precisión involucrada en el intercambio (digamos) no es importante, pero puede serlo para otro fin.

El primer tipo de variablilidad es una fuente material de variabilidad. Este primer tipo de variabilidad se representa en el diagrama por los círculos pequeños que también representan los dominios de las subtécnicas. Las técnicas pueden ser las mismas desde el punto de vista de la función $F$ de $T$, pero pueden variar en la manera como están materialmente constituidas. Por ejemplo, las aleaciones de las que están hechos ciertos instrumentos, o los mecanismos utilizados en la construcción de detectores pueden incluir diferentes materiales y partes, y estas variaciones son una importante fuente de variabilidad. Un segundo tipo de variación agregativa consiste en la multiplicidad de técnicas que pueden utilizarse en un nodo $x$ (que si bien son equivalentes con respecto a $F$ no lo son necesariamente con respecto a otra función de otra técnica diferente de $T$ ). Este es el caso en el que en el contexto de la técnica $T$ sea posible en el paso $x$ la utilización de diferentes técnicas, no sólo materialmente diferentes, sino diferentes en el tipo de proceso o procedimiento que utilizan.

Un ejemplo del segundo tipo de variabilidad se encuentra en las llamadas técnicas de hibridación de ácidos nucléicos. ${ }^{9}$ Estas técnicas se han atrincherado en una gran variedad de técnicas, i.e., entran como subtécnicas en muchas otras técnicas. Nos permiten medir la proporción de hibridación entre dos moléculas. Por ejemplo, la reacción puede medirse: (a) midiendo la absorción de rayos ultravioleta de la muestra; (b) midiendo la proporción de moléculas híbridas que quedan atrapadas en una columna de hidroxiapatita; o (c) graficando el cambio de temperatura requerido para disociar.

presente. $A$ puede depender de $B$ en el sentido de que si $B$ tiene lugar de manera defectuosa entonces $A$ no tiene el resultado deseado. $A$ puede depender de $B$ en el sentido que si $A$ tiene lugar de manera óptima entonces $B$ tiene lugar, pero si $A$ resulta en un paso ligeramente defectuoso esto ya es suficiente para que $B$ no tenga un resultado aceptable (dentro de los márgenes de error asociados con un cierto fenómeno). Es importante estudiar estos y otros sentidos de dependencia, pero su estudio no es importante para el desarrollo del tema de este trabajo.

9 Ésta es una técnica originaria de la biología molecular que cumple diversas funciones en la estabilización de diferentes tipos de fenómenos (véase Martínez y Suárez, 1996). 
diferentes muestras de ácidos hibridizados obtenidas en diferentes momentos de la reacción. Otros tipos de variabilidad se presentan en Martínez y Suárez, 1996.

Nótese que en la caracterización de los tipos de variabilidad es muy importante el hecho de que la variabilidad es agregativa con respecto a la función en cuestión. Es la agregatividad la que permite la localización (ya sea en un paso, o en un módulo de la técnica $T$ ) de la variación de manera que puede ser sometida eficientemente a un proceso de selección. No es necesario que toda la técnica, o muchos de sus pasos, cambien de una sola vez. Es más, de ocurrir esto, lo más probable es que la adecuación de la técnica se vea disminuida seriamente. Las variaciones pueden ser muy pequeñas y no perturbar la función original de la técnica.

Esta breve presentación de la manera como puede modelarse el proceso de la construcción de técnicas y fenómenos nos permite hacer ver cómo el modelo sustenta una explicación causal seleccionista de ese proceso. A diferencia de los modelos tradicionales de epistemología evolucionista, en el modelo que aquí se presenta se especifica en qué consiste el proceso de la replicación de variantes de manera tal que puede verse la forma en que los mecanismos de generación de variantes están asociados con los mecanismos de selección y de heredabilidad de las características que nos permiten hablar de evolución.

\section{Las implicaciones para un proyecto de la naturalización de la epistemología}

No es suficiente el hecho de reconocer que la mente humana, y por lo tanto, el conocimiento científico, son el producto de la evolución biológica. Es necesario reconocer las implicaciones que este hecho tiene para nuestras teorías de la ciencia en más de una dimensión. Muchas veces se piensa que el reconocimiento de que nuestra mente es el producto de la evolución puede ponerse al servicio de una filosofía de la ciencia no-naturalista. Se piensa que la naturalización de la epistemología no requiere cambios profundos en nuestras categorías epistémicas fundamentales, sino que puede asimilarse en las categorías tradicionales por medio de pequeños cambios relativamente superficiales. Popper, por ejemplo, trata de justificar la tesis de que el cambio de teorías es un proceso evolutivo, mediante la analogía entre órganos y teorías (1972, p. 145). ${ }^{10}$ Sin embargo, claramente existen importantes diferencias en el tipo de procesos que dan lugar a los órganos en organismos biológicos, y los que dan lugar a las teorías. Los órganos son el

10 Más en general, una idea muy extendida, formulada explícitamente por Campbell en 1964, y por varios autores, incluido Popper posteriormente, es la idea de que todo conocimiento puede modelarse como el resultado de un proceso de ensayo y error. 
producto de evolución filogenética, mientras que las teorías son el producto de procesos ontogenéticos. Hay muchas otras diferencias significativas que pueden señalarse (véanse por ejemplo los trabajos de Thagard y Cordero, en Martínez y Olivé, 1996).

Un problema central de los modelos evolucionistas del crecimiento del conocimiento es la necesidad de explicar el sentido en el que una variación es "ciega". Es muy difícil justificar una analogía con la evolución orgánica acorde con los lineamientos de la teoría de la evolución de Darwin si no existe un sentido claro en el que la variación en cuestión sea ciega, o una explicación satisfactoria de por qué no tiene que ser ciega. Como muchos críticos de los modelos evolucionistas han hecho ver (véanse los artículos de Brady, Thagard y Cordero en Martínez y Olivé, 1996), la búsqueda de conjeturas, por ensayo y error, no es ciega. Otro aspecto en el que la analogía pareçe particularmente débil tiene que ver con el hecho de que en la naturaleza no hay intencionalidad, mientras que en la ciencia la intencionalidad es un aspecto central.

Si pensamos en las teorías como si fueran análogas a los organismos, esas críticas me parecen muy convincentes y definitivas. Las conjeturas y las teorías que se elaboran a partir de ellas no son producidas ciegamente y, lo que es más importante, esta producción no ciega de variantes es parte integral del proceso de elaboración de teorías. Es también claro que si pensamos en teorías como las unidades de cambio, el aspecto intencional involucrado en su elaboración es esencial y, en este sentido (como dice Elster en 1979), hay una "brecha insalvable" entre sistemas biológicos y teorías.

Este tipo de objeciones dejan de tener peso, sin embargo, una vez que enfocamos nuestra atención en unidades evolutivas, que no son meramente construcciones mentales como las teorías, sino que son objetos cuya variabilidad y evolución tienen como base la variabilidad y evolución de restricciones (constraints) materiales que se constituyen en sistemas funcionales por medio de procesos de selección.

Hull (1988, cap. 12) muestra que el hecho de que un proceso involucre agentes intencionales no es una diferencia que no permita modelar ese proceso como un proceso seleccionista. Lo importante para la caracterización de un proceso seleccionista es el tipo de replicadores involucrados en el proceso de selección. Los científicos pueden verse como portadores de teorías (rasgos fenotípicos) y son esos portadores los que están sujetos a la selección. La intencionalidad de estos portadores no juega un papel causal en la explicación, y por lo tanto evade las críticas mencionadas anteriormente. La estrategia que utiliza Hull para evitar las críticas a modelos evolucionistas del conocimiento es muy importante y en general permite a un modelo 
evolucionista como el que aquí presento no tener que preocuparse por desenredar el papel de la intencionalidad en los procesos que se modelan.

Otra objeción a modelos evolucionistas del conocimiento, que tiene su origen en las dificultades que plantea la intencionalidad de las supuestas "unidades de evolución", es la siguiente: un organismo contribuye a la sobrevivencia de su linaje en relación directa con su capacidad de reproducirse en diferentes entornos, una capacidad que se mide en términos de "adecuación". Un modelo evolucionista que no permita una caracterización mínimamente precisa del concepto de adecuación difícilmente puede considerarse un modelo evolucionista. Como dice Brady (en el artículo incluido en Martínez y Olivé, 1996): la verdad no puede funcionar como el correlato de la adecuación. No sólo porque la verdad de teorías no acepta grados, sino porque la verdad no es una propiedad relacional como la adecuación. La analogía se rompe, dice Brady, porque en la evolución orgánica hay, en última instancia, un único problema, en la evolución conceptual hay muchos. Esto es lo que haría difícil la caracterización de criterios de selección conceptual asociados con una medida de adecuación, algo que es necesario para hacer de un proceso evolucionista del conocimiento algo más que una metáfora. Como hemos visto, en el caso del modelo de la evolución de técnicas y fenómenos que presento aquí, hay un concepto claro de adecuación que es totalmente análogo al concepto clásico de adecuación. La adecuación de una técnica $T$ es una medida de su capacidad de reproducirse en los diferentes entornos (aplicaciones) accesibles en un momento dado. Por ello, indirectamente, el atrincheramiento y la robustez de la técnica son buenos indicadores de su adecuación (suponiendo que no hay cambios fuertes en los entornos accesibles).

En el caso del modelo evolucionista de técnicas que propongo, esta objeción de Brady puede responderse como sigue: en primer lugar, no es cierto que la evolución orgánica sea en última instancia un solo problema, por lo menos en el sentido pertinente. Hay procesos de evolución que tienen lugar en el sistema inmunológico, en la organización neuronal y en muchos otros procesos biológicos. Estos procesos son, en importantes sentidos, relativamente independientes y diferentes del proceso de evolución orgánica. La diversidad de procesos cognoscitivos modelables por medio de procesos evolutivos puede verse en analogía con ese tipo de variedad de procesos evolutivos que tiene lugar en el nivel biológico. En segundo lugar, como hemos visto, en el caso del modelo de la evolución de técnicas y conceptos aquí presentado puede formularse un concepto claro de adecuación. Una propuesta similar para un modelo como el de Campbell no es posible porque no hay manera de hacer claro el concepto mismo de replicación. Hay muchas maneras posibles en las que podemos decir que una idea 
se replica, y cada una de estas maneras daría una medida de adecuación diferente. En el caso de las técnicas la idea puede hacerse precisa porque a fin de cuentas la adecuación se refiere a propiedades de sistemas materiales que son el resultado de procesos causales, que hacen del mecanismo de variación un proceso que permite sustentar una explicación seleccionista.

\section{Conclusión}

El desafío de Campbell consiste en exigir que se especifique el tipo de conocimiento que necesita explicarse por medio de los modelos evolucionistas del conocimiento. La respuesta de Campbell no sólo deja una brecha significativa entre lo que el modelo puede explicar y la historia del cambio de teorías, sino que presupone un realismo de los entes teóricos que es muy cuestionable. La propuesta alternativa que he sugerido en este trabajo es que un tipo de conocimiento que se presta a una explicación por medio de modelos evolucionistas no es el conocimiento sistematizado en teorías, sino el conocimiento que se genera en las tradiciones experimentales. Cuestiones acerca del cambio conceptual en teorías muy probablemente requieren consideraciones que escapan a un modelo evolucionista del cambio conceptual en la ciencia. Esto sólo puede verse como una objeción a mi respuesta al desafío de Campbell si se piensa que el problema del cambio científico es el problema del cambio de teorías. Pero en realidad el problema.del cambio científico es una serie de problemas con parentescos a veces lejanos.

BIBLIOGRAFIA

Campbell, Donald, 1974, "Evolutionary Epistemology", en Schlipp P. (comp.), The Philosophy of Karl Popper.

—, 1990, "Epistemological Roles for Selection Theory", en Nicholas Rescher (ed.), Evolution, Cognition and Realism, Studies in Evolutionary Epistemology, University Press of America, Lanham.

Cartwright, Nancy, 1983, How the Laws of Physics Lie, Oxford University Press, Oxford.

Cherniak, C., 1986, Minimal Rationality, A Bradford Book, The MIT Press, Cambridge Massachusetts, Londres.

Daston, L., 1988, Probabilities in the Enlightment, Harvard University Press.

Edelman, G., 1987, Neural Darwinism, The Theory of Neuronal Group Selection, Basic Books.

Elster, 1989, Salomonic Judgements: Studies in the Limitation of Rationality, Cambridge University Press, Cambridge.

Feyman, 1965, The Character of Physical Law, MIP Press, Cambridge Massachusetts. 
Gallison, P., 1987, How Experiments End, The University of Chicago Press, Chicago. Hacking, 1983, Representing and Intervening, Cambridge University Press, Cambridge. [Trad. castellana S. Martínez, Instituto de Investigaciones Filosóficas de la UNAM (en prensa en coedición con Paidós).]

Holland, J., 1986, Holyak K., R. Nisbett y P. Thagard, Induction, Processes of Learning and Discovery, The MIT Press.

Hull, D., 1988, Science as a Process, The University of Chicago Press, Chicago.

Kahneman, D., Slovic P. y A. Tversky, 1982, Judgement under Uncertainty: Heuristics and Biases, Cambridge University Press, Cambridge.

Laudan, L., 1984, Science and Values, University of California Press.

Martínez S., 1990, "Mas allá de la presuposición newtoniana: propiedades genuinamente disposicionales en la mecánica cuántica", Crítica, vol. XXII, no. 66, 1990, pp. 25-37.

_-, 1993, "Método, evolución y progreso en la ciencia", Crítica, (primera parte), vol. XXV, no. 73, 1993, pp. 37-69; Crítica, (segunda parte), vol XXV, no 74, 1993, pp. 3-20.

__, 1995a, "La autonomía de las tradiciones experimentales como problema epistemológico", Crítica, vol. XXVII, no. 80, 1995, pp. 3-48.

- $1995 \mathrm{~b}$, "The Evolution of Material Constraints in the Structure of Scientific Knowledge", (manuscrito).

Martínez, S. y L. Olivé (comps.), Epistemología y evolución, Seminario de Problemas Científicos y Filosóficos de la UNAM, Paidós-UNAM (en prensa).

Martínez, S. y E. Suárez, 1996, "La evolución de técnicas y fenómenos: hacia una explicación de la 'confección' del mundo", Crítica, vol. XVIII, no. 82, 1996, pp. 2566.

Pickering, A., 1984, Constructing Quarks, The University of Chicago Press, Chicago. The University of Edinburgh Press, Edimburgo.

Popper, 1972, Objective Knowledge, Clarendon Press, Oxford.

Shapin, S. y S. Schaffer, 1985, Leviathan and the Air Pump, Hobbes, Boyle and the Experimental Life, University Press, Princeton.

Wimsatt, 1980, "Reductionistic Research Strategies and their Biases in the Units of Selection Controversy", en T. Nickles (comp.), Scientific Discovery, vol. 2, Case Studies, D. Reidel, Dordrecht.

_ - 1986a, "Heuristics and the Study of Human Behavior", en D.W. Fiske y R. Shweder (comps.), Metatheory in Social Science: Pluralism and Subjectivities, University of Chicago Press, Chicago, pp. 293-314.

—_, 1986b, "Forms of Aggregativity", en A. Donagan, A.N. Perovich (Jr.), y M.V. Wedin (eds.), Human Nature and Natural Knowledge. 